\title{
Vitamin E supplementation during the dry period in dairy cattle. Part II: Oxidative stress following vitamin E supplementation may increase clinical mastitis incidence postpartum
}

\author{
R. J. Bouwstra, ${ }^{\star 1}$ M. Nielen, ${ }^{*}$ J. R. Newbold,† E. H. J. M. Jansen, $\neq$ H. F. Jelinek,§ and T. van Werven \\ *Faculty Veterinary Medicine, Department of Farm Animal Health, Utrecht University, $3584 \mathrm{CL}$, the Netherlands \\ †Provimi Research and Innovation Centre, 1932 Sint-Stevens-Woluwe, Brussels, Belgium \\ $\ddagger$ National Institute for Public Health and the Environment, 3721 MA Bilthoven, the Netherlands \\ §School of Community Health, Centre for Research in Complex Systems, Albury, New South Wales 2640, Australia
}

\begin{abstract}
The aim of this study was to evaluate, retrospectively, which physiological states influenced the effect of vitamin E supplements during the dry period on the level of oxidative stress at 2 wh antepartum. Furthermore the effect of oxidative stress at 2 wk antepartum on the risk of clinical mastitis in early lactation was investigated. Cows experience oxidative stress around calving. Vitamin $\mathrm{E}$ is able to decrease oxidative stress by scavenging free radicals. Normally, vitamin E radicals formed when vitamin $\mathrm{E}$ reacts with free radicals are regenerated by a network of other antioxidants, termed the "vitamin E regeneration system" (VERS). In case of vitamin E supplementation, VERS should be sufficient to regenerate formed vitamin E radicals; if not, oxidative stress might increase instead of decrease. Additionally, the level of oxidative stress and vitamin $\mathrm{E}$ might be important physiological states to evaluate before supplementation. In a clinical trial, 296 cows on 5 farms were randomly divided into 2 groups, supplemented with a mineral mix between dry off and calving that supplied 3,000 or $135 \mathrm{IU} / \mathrm{d}$, respectively. Blood samples collected at dry off and 2 wk antepartum were analyzed for vitamin E, reactive oxygen metabolites, ferric-reducing ability of plasma, glutathione peroxidase, and malondialdehyde. Cows were allocated retrospectively into 8 subgroups based on the level of oxidative stress, vitamin E, and VERS status at dry off. To evaluate whether differences in physiological states at dry off influenced the effect of vitamin E supplementation on the level of oxidative stress, group effects (supplemented vs. control) were studied with Student's $t$-test for all 8 subgroup at $2 \mathrm{wk}$ antepartum. Differences in physiological states at dry off influenced the effect of vitamin E supplements. In 2 insufficient VERS subgroups, the supplemented group had higher levels of free radicals at 2 wk antepartum
\end{abstract}

Received February 10, 2010.

Accepted September 12, 2010.

${ }^{1}$ Corresponding author: r.j.bouwstra@uu.nl compared with the control group. Relative risk calculation was used to study the effect of oxidative stress at 2 wk antepartum on the incidence of mastitis in the first $100 \mathrm{~d}$ of lactation. Higher levels of oxidative stress at $2 \mathrm{wk}$ antepartum were related to higher risk of clinical mastitis. In conclusion, not every dry cow responded well to high vitamin E supplementation. This subgroup analysis provides a possible explanation for the unexpected adverse effects observed in the clinical trial.

Key words: dairy dry cow, mastitis, oxidative stress, vitamin $\mathrm{E}$

\section{INTRODUCTION}

Reported effects of vitamin E supplementation during the periparturient period on subsequent udder health of dairy cows have been inconsistent. Several studies showed positive effects (Smith et al., 1984; Weiss et al., 1990a), whereas more recent studies showed no effects (LeBlanc et al., 2004; Persson Waller et al., 2007). Furthermore, several reports have described adverse effects of vitamin E supplements on human health (Miller et al., 2005; Brigelius-Flohé, 2007; Winterbone et al., 2007). Recently, we observed an unexpected adverse effect of vitamin E supplementation on (sub)clinical mastitis incidence in a large, randomized, double-blind clinical trial (R. J. Bouwstra, M. Nielen, J. R. Newbold, E. H. J. M. Jansen, H. F. Jelinek, and T. van Werven, unpublished data). These unexpected and inconsistent results raised the need to rethink the mechanisms of antioxidant activity of vitamin $\mathrm{E}$ in dairy cows.

During the periparturient period, dairy cows experience oxidative stress (Brzezinska-Slebodzinska et al., 1994; Bernabucci et al., 2005; Castillo et al., 2005), which develops when free radical generation exceeds antioxidant capacity (Miller et al., 1993). Vitamin E ( $\alpha$-tocopherol), the primary lipid-soluble antioxidant, is important for the body's defense against oxidative stress (Ibrahim et al., 1997), neutralizing free radicals by formation of the prooxidant $\alpha$-tocopheroxyl radical. Under normal physiological states, this prooxidant 


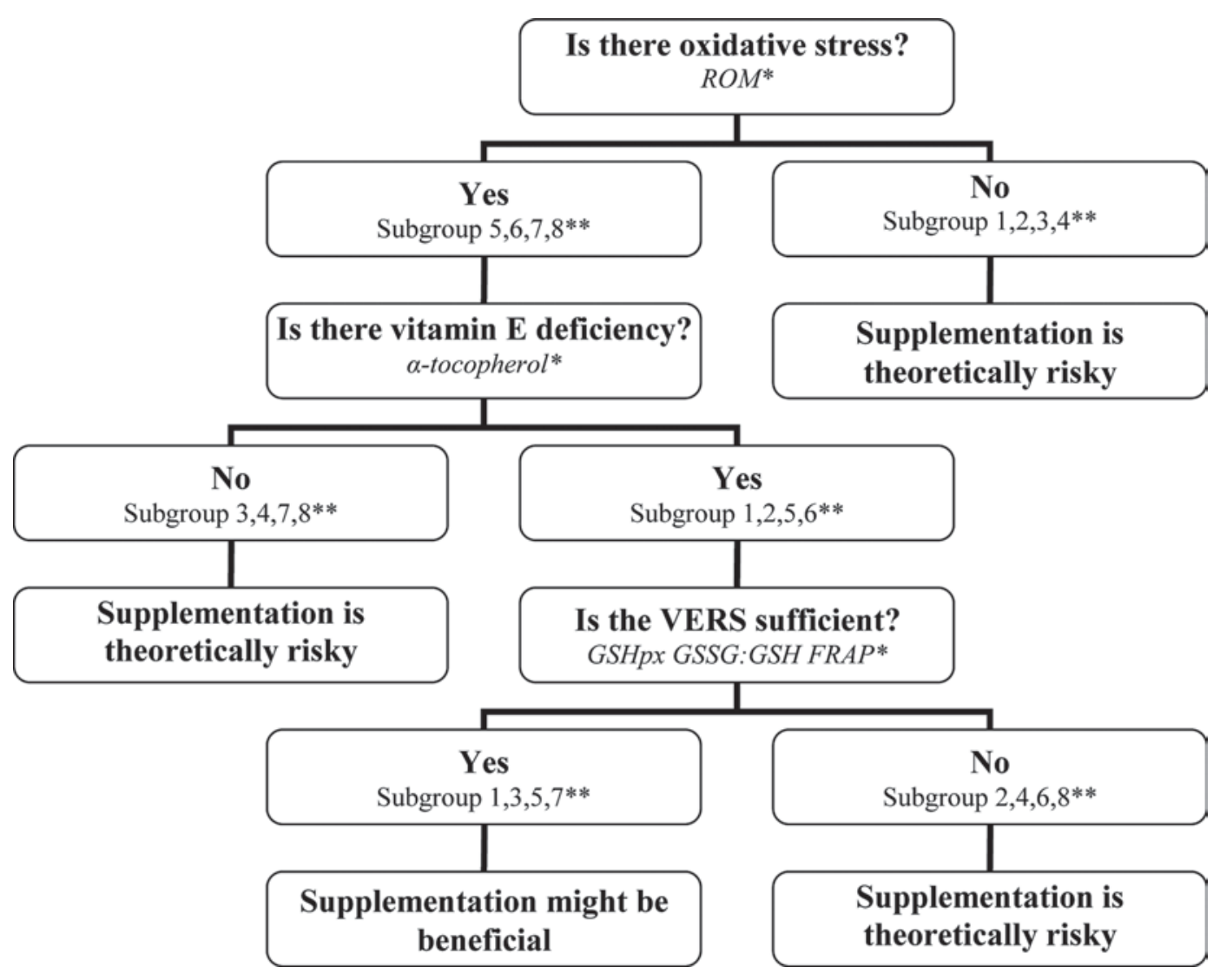

Figure 1. Hypothetical decision tree to assess the effect of vitamin E supplementation adapted from Nwose et al. (2008). *Blood parameters measured in the present study; ${ }^{* *}$ subgroup classification as mentioned in Table $1 . \mathrm{ROM}=$ reactive oxygen metabolites; GSHpx $=$ glutathione peroxidase; GSSG:GSH = ratio of oxidized glutathione to reduced glutathione; FRAP = ferric-reducing ability of plasma assay.

will be regenerated to $\alpha$-tocopherol by an antioxidant network of enzymatic and nonenzymatic reactions. Among others, glutathione peroxidase (GSHpx) and vitamin $\mathrm{C}$ are important interacting co-antioxidants to neutralize the $\alpha$-tocopheroxyl radical (Frei et al., 1988; Ho and Chan, 1992; Young and Woodside, 2001). All complex interactions together needed to regenerate $\alpha$-tocopheroxyl radicals have been called the "vitamin E regeneration system" (VERS) (Nwose et al., 2008). When animals and humans are supplemented with vitamin E, VERS should be sufficient to regenerate or reduce $\alpha$-tocopheroxyl radicals back to $\alpha$-tocopherol. $\alpha$-Tocopheroxyl radicals are regenerated by the activity of reduced glutathione (GSH), vitamin C, and Q10. During oxidative stress mechanisms, the amount of GSH, vitamin C, and Q10 available for regeneration of $\alpha$-tocopherol is reduced and a build up of the $\alpha$-tocopherol free radical occurs, which might lead to oxidative stress-related damage (Young and Woodside, 2001). With potential adverse effects of vitamin E supplementation, prior physiological states for effective use of additional vitamin E need to be investigated. Based on the hypothesis of Nwose et al. (2008), we propose 3 important prior physiological states: oxidative stress, vitamin E status, and VERS. We include VERS here as a hypothesis that incorporates the main molecules that play a role in balancing $\alpha$-tocopherol/ $\alpha$-tocopheroxyl radicals

First, no oxidative stress before vitamin E supplementation might be a contraindication for extra vitamin E supplements. Second, vitamin E levels before supplementation must not be too high. Third VERS must be sufficient. Under this hypothesis, vitamin E supplementation will only be effective in reducing existing oxidative stress given oxidative stress, a low level of vitamin E, and sufficient VERS; under other physiological states, it might be neutral or detrimental (Figure 1).

In this study, the 3 physiological states oxidative stress, vitamin E and VERS status before supplementation were based on blood levels of reactive oxygen metabolites (ROM), an indicator of free radical production (Yamanaka et al., 2006); $\alpha$-tocopherol; and reduced GSH and oxidized glutathione (GSSG), the 
enzymatic antioxidant GSHpx, and the ferric-reducing ability of plasma assay (FRAP). Glutathione peroxidase represents the main form of intracellular antioxidant defense (Sen et al., 1994). The FRAP offers an index of antioxidant potential of biological fluids and can be used to assess the antioxidant power of serum (Benzie and Strain, 1996). The level of FRAP is influenced by the level of vitamin $\mathrm{C}$, uric acid, bilirubin, total protein, and albumin, all of which are able to work as co-antioxidants. The GSSG:GSH ratio, as well as GSHpx and FRAP, are an essential part of our explanatory hypothesis, which is based on $\alpha$-tocopherol being oxidized to $\alpha$-tocopheroxyl radical and being reduced by GSH. The GSH is in turn oxidized to GSSG, which is then back-converted to reduced GSH by vitamin C. Therefore, we chose these substances as experimental indicators of VERS. Abnormal levels of GSSG:GSH, GSHpx, and FRAP in our study were based on the human literature. We selected the 80th percentile for GSSG:GSH and the 20th percentile for GSHpx and FRAP to define oxidative stress and an imbalance of the VERS (Nwose et al., 2008).

The aim of this study was to evaluate retrospectively whether 3 physiological states before supplementation influence the effect of vitamin E supplementation during the dry period on the level of free radicals and oxidative damage at 2 wk antepartum in dairy cows. Furthermore the relation between the level of free radicals and oxidative damage at $2 \mathrm{wk}$ antepartum on the risk of clinical mastitis in early lactation was investigated. Free radicals and oxidative damage $2 \mathrm{wk}$ antepartum were measured as ROM and malondialdehyde (MDA), a degradation product of lipid peroxidation (Nielsen et al., 1997).

\section{MATERIALS AND METHODS}

This study used results from an experiment conducted in the Netherlands that followed an experimental protocol approved by The Committee for Animal Experiments of Utrecht University (see Bouwstra et al., 2010 for more details).

\section{Animals}

The experiment was carried out from August 2007 to July 2008 with 296 Holstein Friesian dairy cows in 5 commercial dairy herds in the Netherlands (see the companion paper for more details; Bouwstra et al., 2010). On each farm, 52 to 70 cows were selected and randomly divided over the 2 experimental groups. On 3 farms (B, D, E), heifers were included in the experiment as a separately randomized group. The cows and heifers in the control group $(\mathrm{n}=148)$ received a daily mineral mix providing $135 \mathrm{IU}$ of vitamin $\mathrm{E} / \mathrm{d}$, and cows and heifers in the supplemented group $(\mathrm{n}=148)$ received a daily mineral mix providing 3,000 IU of vitamin $\mathrm{E} / \mathrm{d}$ (Provimi BV, Rotterdam, the Netherlands). Other vitamins and minerals in the dry cow mineral mixes were equal and met Dutch standards for dry cow supplements. Cows were dried off at 8 wk before expected calving, and the mineral mix was fed during the entire dry period.

\section{Blood Sampling}

All cows were sampled at dry off and 2 wk before the expected calving day. All samples were collected between 8 and 12 a.m. Samples were kept at 6 to $10^{\circ} \mathrm{C}$ before they arrived at the laboratory. Mean time from sampling until arrival in the laboratory was $3 \mathrm{~h}$. Blood was collected from the jugular vein with BD Vacutainer systems (Becton Dickinson, Plymouth, UK). Blood samples were collected in an SST II Advance tube (Becton Dickinson). Serum samples were centrifuged for $15 \mathrm{~min}$ at $4,465 \mathrm{rpm}(3,500 \times g)$, at $4^{\circ} \mathrm{C}$. Heparinized samples were centrifuged $(10 \mathrm{~min}$ at $2,000 \times g$ and $4^{\circ} \mathrm{C}$ ) and washed in PBS 3 times immediately after collection to collect erythrocytes. All samples were frozen at $-80^{\circ} \mathrm{C}$ until analysis. Serum was analyzed for vitamin E, MDA, ROM, and FRAP levels. Erythrocytes were analyzed for GSH-Px activity, GSH, and GSSG concentration.

\section{Definition of Mastitis}

Cases of mastitis were defined by the farmer and based on abnormalities visible in the cow, in milk, or both between 0 and $100 \mathrm{~d}$ postpartum. Abnormalities defined for the cow were a listless cow with or without an abnormal body temperature in combination (or not) with reduced appetite, always related to any abnormalities in the udder or milk. Abnormalities defined for the udder were a red, swollen, and possibly painful udder. Abnormalities defined for the milk were watery milk and milk containing clots and possibly pus. When a farmer noticed a clinical quarter case, he or she would contact the emergency service of the research team directly. Within $24 \mathrm{~h}$, the farm with the diseased cow was visited by a veterinarian for diagnosis of clinical mastitis. Farmers and all employees of the farms were trained by one veterinarian to recognize new clinical cases. Cows with and without clinical mastitis were used to investigate the relationship between the level of $\log \mathrm{ROM}$ and $\log \mathrm{MDA}$ at 2 wk antepartum and clinical mastitis. 


\section{Laboratory Analysis}

The concentration of hydroperoxides was assessed by a spectrophotometric test (the d-ROM) wherein a small amount of serum was diluted in an acidic solution ( $\mathrm{pH}$ 4.8). In these physiological states, iron ions become available to catalyze the breakdown of hydroperoxides to alkoxyl and peroxyl radicals. The chromogenic substrate used was $N, N$,-dimethyl-p-phenylenediamine, which is transformed into a pink- to red-colored radical cation. The concentration of colored complex is directly related to the hydroperoxide levels of the tested biological sample.

Vitamin E ( $\alpha$-tocopherol) was measured by an isocratic HPLC method using a kit from Chromsystems (Munich, Germany). The kit contained reagents, internal standard, calibration standards, an HPLC column, and mobile phase. The HPLC equipment comprised components from Varian (Middelburg, the Netherlands). Detection occurred with fluorescence detection.

Analyses for GSH, GSSG, and hemoglobin were performed routinely on a clinical autoanalyzer (Hitachi 912, Roche Diagnostics, Almere, the Netherlands) using commercial kits (Roche Diagnostics). The GSH-Px activity was measured on the same autoanalyzer with kits from Randox (Antrim, UK). The MDA was measured in HPLC by fluorescence detection using a kit from Chromsystems. The FRAP antioxidant assay was measured using the method described by Benzie and Strain (1996). The FRAP assay uses a low-pH reductant to reduce ferric tripyridyltriazine [Fe(III)-TPTZ] complex to ferrous tripyridyltriazine [Fe(II)-TPTZ]. Ferrous tripyridyltriazine has an intense blue color and can be monitored at $593 \mathrm{~nm}$ (Benzie and Strain, 1996). The relative activities of samples were assessed by comparing their activities with Trolox. This assay offers an index of the antioxidant power of serum.

All analyses were performed at the National Institute for Public Health and the Environment (Bilthoven, the Netherlands).

\section{Statistical Analysis}

General. For all analyses, vitamin E, MDA, ROM, and FRAP data were log-transformed because the residuals looked more normally distributed. The overall effect of oral vitamin E supplementation on the level of vitamin $\mathrm{E}$ ( $\alpha$-tocopherol), free radicals (ROM), oxidative damage (MDA), and antioxidant capacity of serum (FRAP) at 2 wk antepartum was analyzed with Student's $t$-test and in mixed models. A mixed model with group, time, parity, and group $\times$ time interaction as fixed effects, and cow, time, farm, and time and cow within farm as random effects was fitted using REML for the random effects and ML for the fixed effects. The difference between groups during the dry period was tested with a group $\times$ time interaction effect. To test the group $\times$ time interaction effect, vitamin $\mathrm{E}, \mathrm{MDA}$, ROM, and FRAP levels at 2 wk antepartum were tested with reference to the first measurement at dry off. All models were built in $\mathrm{R}$ (v. 2.11.0, R Foundation for Statistical Computing, Vienna, Austria) and Student's $t$-tests were carried out in SPSS 12.0.1 for Windows.

Subgroups. Retrospectively, cows were divided into different subgroups based on the 3 physiological states: oxidative stress, vitamin E status, and VERS at dry off (T0). The physiological state "oxidative stress" was based on the level of ROM, and the physiological state "vitamin E status high" was based on the level of $\alpha$-tocopherol. The physiological state "VERS" was based on the ratio of reduced and oxidized glutathione, the level of the enzymatic antioxidant GSHpx, and FRAP. To calculate with the 3 physiological states (oxidative stress, vitamin E status, and VERS), the median values of ROM $(39.7 \mathrm{U} / \mathrm{mL})$ and vitamin $\mathrm{E}(15.8 \mu \mathrm{mol} / \mathrm{L})$, the 20th percentiles for FRAP and GSHpx, and the 80th percentile for the GSH:GSSG ratio at T0 were used as cut-off values. The VERS was defined as sufficient for the GSSG:GSH ratio $\leq 0.87$ (80th percentile in frequency distribution at T0), the level of GSHpx $>3.42$ $\mathrm{U} / \mathrm{L}$ (20th percentile in frequency distribution at T0), and FRAP $>222 \mu \mathrm{mol} / \mathrm{L}$ (20th percentile in frequency distribution at T0). Any other combination of these 3 parameters was defined as an insufficient VERS. Cut-off points were data driven because no biological support was available to set high or low levels of ROM, FRAP, and GSSG:GSH for cows. Corresponding to the 8 possible combinations of oxidative stress, vitamin $\mathrm{E}$, and VERS status, 8 subgroups of cows at dry off were formed (Table 1 ). Over these 8 subgroups, cows received high (3,000 IU/d; supplemented group) or low (135 IU/d; control group) vitamin $\mathrm{E}$ amounts during the dry period; cows were not randomized within the 8 subgroups because these subgroups were defined retrospectively. Within the 8 different subgroups, the effect of vitamin E supplementation on free radical levels and oxidative damage was assessed in terms of the difference in ROM and MDA between the supplemented and control group at 2 wk antepartum. To evaluate whether differences in the 3 physiological states at T0 influenced the effect of vitamin E supplementation on the level of ROM and MDA at 2 wk antepartum, group effects (supplemented vs. control) were studied in 2 steps. Visual inspection of box plots of ROM and MDA in each of the 8 subgroups indicated whether ROM or MDA differed between the 2 vitamin $\mathrm{E}$ treatment groups. In addition to the visual inspection, a Student's $t$-test was carried out on ROM and MDA levels at 2 wk antepartum for each subgroup 
Table 1. The 8 different subgroups that were retrospectively based on the 3 physiological states: oxidative stress, vitamin E status, and vitamin E regeneration system (VERS) measured at $\mathrm{T}^{1}$

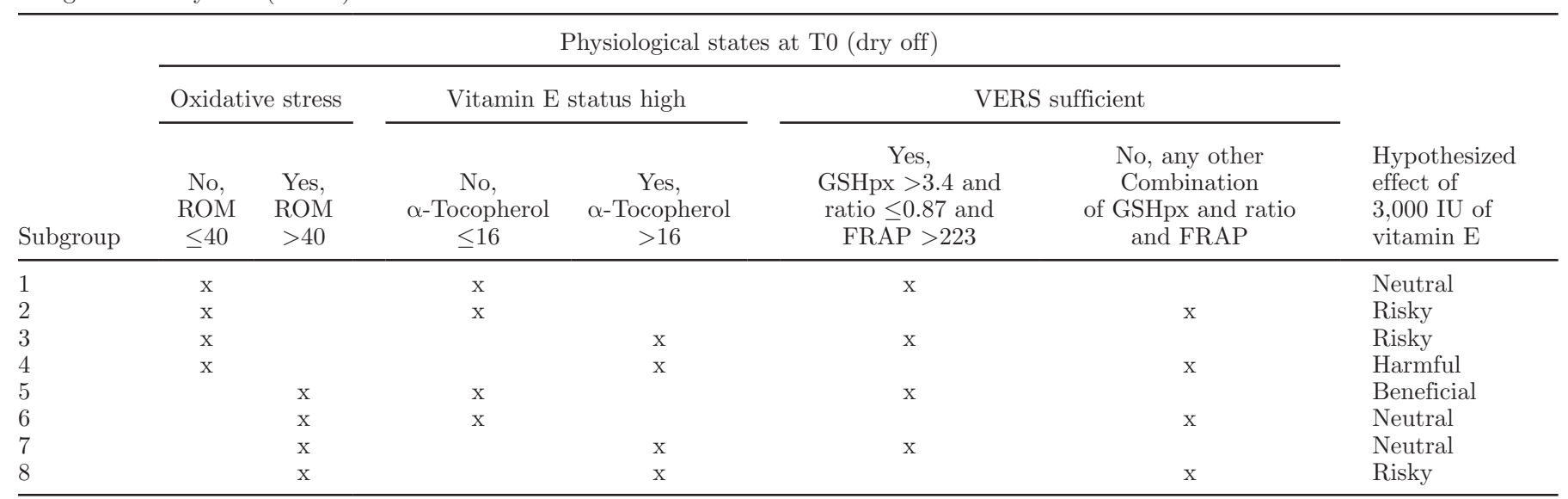

${ }^{1} \mathrm{ROM}=$ reactive oxygen metabolites; FRAP $=$ ferric-reducing ability of plasma assay; GSHpx $=$ glutathione peroxidase.

(SPSS 12.0.1 for Windows) potentially to support visual trends. Consequently, Student's $t$-test $P$-values $<$ 0.1 are reported to indicate these possible trends.

Clinical Mastitis. Healthy cows and cows that developed clinical mastitis before 100 DIM were compared in terms of ROM and MDA levels with Student's $t$-test separately in the supplemented and control group at 2 wk antepartum. Student's $t$-tests were carried out in SPSS 12.0.1 for Windows.

The relative risks of high levels of ROM or MDA at 2 wk antepartum on clinical mastitis were calculated using a cohort analysis. A ROM level of $>52.2 \mathrm{U} / \mathrm{mL}$ and a MDA level $>0.26 \mu \mathrm{g} / \mathrm{L}$ were defined as high at 2 wk antepartum. Both values are in the 80 th percentile in frequency distribution at $2 \mathrm{wk}$ antepartum, again by lack of biological support to set high or low levels for dry cows. Relative risk analyses were analyzed with Win Episcope 2.0 (Thrusfield et al., 2001).

\section{RESULTS}

\section{General}

Differences for $\alpha$-tocopherol, FRAP, MDA and ROM between the supplemented and control group and time trends are summarized in Table 2 and Figure 2. At 2 wk antepartum, vitamin E concentration was higher in the supplemented group compared with the control group (group effect). In both groups (supplemented and control), vitamin $\mathrm{E}$ levels were lower at 2 wk antepartum compared with T0 (time effect), but vitamin E levels decreased less in the supplemented group (group $\times$ time effect). High vitamin $\mathrm{E}$ amounts increased the antioxidant capacity of serum as FRAP levels were also higher in the supplemented group at 2 wk antepartum (group effect). The FRAP levels decreased during the dry period in both groups (time effect), but decreased less in the supplemented group (group $\times$ time effect). For ROM and MDA, neither group differences at 2 wk antepartum nor time or group $\times$ time effects were observed relative to dry off.

\section{Subgroups}

For MDA and ROM, the differences between the supplemented and control group at 2 wk antepartum within the 8 subgroups are summarized in Table 3 and Figure 3. In contrast to the overall results, differences between the supplemented and control group were found at 2 wk antepartum for ROM in subgroups 4 and 6 , and for MDA in subgroup 3. In cows without oxidative stress, a high vitamin E status, and an insufficient VERS at T0 (subgroup 4), and in cows with oxidative stress, without a high vitamin E status, and an insufficient VERS at T0 (subgroup 6), supplementation of $3,000 \mathrm{IU}$ of vitamin $\mathrm{E}$ tended to increase the level of ROM, indicating increased free radicals $(P=0.059$ and $P=0.010$, respectively). In none of the subgroups did supplementation with 3,000 IU of vitamin E/d lead to significantly lower ROM levels at 2 wk antepartum. In cows without oxidative stress, a high vitamin E status, and a sufficient VERS at T0 (subgroup 3), supplementation of 3,000 IU of vitamin $\mathrm{E}$ decreased the level of MDA $(P=0.05)$, indicating lower levels of lipid peroxidation in the supplemented group.

\section{Clinical Mastitis}

The ROM and MDA levels at 2 wk antepartum in cows that developed clinical mastitis and in healthy cows are summarized in Table 4 . In cows supplemented with vitamin E, ROM and MDA levels at 2 wk an- 
Table 2. Results of the Student's $t$-tests between the high and low supplemented group at 2 wk antepartum and results of 4 linear mixed models for the dependent variables $\alpha$-tocopherol, ferric-reducing ability of plasma assay (FRAP), malondialdehyde (MDA), and reactive oxygen metabolites (ROM) with group, time, parity, and group $\times$ time interaction as fixed effects, and cow, time, farm, and time and cow within farm as random effects $^{1}$

\begin{tabular}{lclcl}
\hline Variable & $\begin{array}{c}\text { Group } \\
t \text {-test }\end{array}$ & $\begin{array}{l}\text { Level in the high } \\
\text { group at 2 wk antepartum }\end{array}$ & $\begin{array}{c}\text { Group } \times \text { time } \\
\text { mixed models }\end{array}$ & $\begin{array}{l}\text { Direction over time } \\
\text { in both groups }\end{array}$ \\
\hline$\alpha$-Tocopherol & 0.000 & Higher & 0.000 & Decreasing \\
FRAP & 0.003 & Higher & 0.002 & Decreasing \\
MDA & 0.985 & - & 0.998 & - \\
ROM & 0.306 & - & 0.292 & - \\
\hline
\end{tabular}

${ }^{1}$ Displayed are the $P$-values of Student's $t$-tests results (group) and $P$-values of group $\times$ time interaction effects of the linear mixed models. Statistical tests were carried out on $\log$ vitamin E, log FRAP, log MDA, and $\log$ ROM values.

tepartum seemed to be higher in cows that went on to develop clinical mastitis than in healthy cows $(P=$ 0.213 and $P=0.144$, respectively).

The relative risk of developing clinical mastitis with high levels of ROM at 2 wk antepartum was $1.2(95 \%$ CI, 0.6-2.4). The relative risk of developing clinical mastitis with high levels of MDA at 2 wk antepartum was 1.6 (95\% CI, 0.9-3.0), and the relative risk of developing clinical mastitis with high levels of both ROM and MDA at 2 wk antepartum was 2.8 (95\% CI, 1.1-7.1).

\section{DISCUSSION}

The results of this study introduce the possibility of risk related to high vitamin $\mathrm{E}$ supplementation without knowing the current status of the individual cow for oxidative stress, vitamin E, and VERS. Supplementation with high vitamin $\mathrm{E}$ amounts (3,000 IU/d) during the dry period to cows with an insufficient VERS at dry off seemed to increase oxidative stress. At $2 \mathrm{wk}$ antepartum, free radical levels in some of those cows (subgroup 4 and 6) were higher compared with cows that received low levels of vitamin $\mathrm{E}(135 \mathrm{IU} / \mathrm{d})$. Oxidative damage at $2 \mathrm{wk}$ antepartum was lower in the supplemented group only in cows without oxidative stress, a high vitamin E status, and a sufficient VERS at dry off (subgroup 3). Additionally, cows with higher levels of free radicals and oxidative damage at $2 \mathrm{wk}$ antepartum had 2 times higher risk of clinical mastitis in early lactation. As it is difficult to evaluate exactly what is cause and effect, additional research is needed to examine the relationship between oxidative stress and disease, such as clinical mastitis.

The results described in this report were retrospectively studied following a clinical trial that showed an adverse effect of vitamin $\mathrm{E}$ in dairy cows (R. J. Bouwstra, M. Nielen, J. R. Newbold, E. H. J. M. Jansen, H. F. Jelinek, and T. van Werven, unpublished data). The unexpected adverse results raised the need to rethink the mechanisms of antioxidant activity of vitamin $\mathrm{E}$ in dairy cows. In human medicine, the toxicity of antioxidant vitamins has been widely reported. The recently published Guidelines of the American Heart Association recommend against vitamin supplements because such supplements may cause harm (Mosca et al., 2007).

In agreement with other dairy research (Goff and Stabel, 1990; Weiss et al., 1990b), vitamin E supplementation in our study increased vitamin E blood concentration. In several studies, vitamin E lowered oxidative stress and in that way improved the health of dairy cows (Miller et al., 1993). Although oxidative stress is often described, in the literature little consensus exists as to how it should be defined. For example, Bernabucci et al. (2005) defined oxidative stress as a result of an imbalance between reactive oxygen metabolites production and the neutralizing capacity of antioxidant mechanisms. Castillo et al. (2005) defined oxidative stress as free radical generation exceeding the body's antioxidant production capacity. The 2 parameters, ROM and MDA, used in the present study give insight into both the level of free radical production and oxidative damage. The d-ROMs test analyzes the concentration of hydroperoxides, which are part of the reactive oxygen species (Yamanaka et al., 2006). Hydroxyperoxides are more polar than parent lipids and can be deleterious to cells by participating in redox reactions (Girotti, 1998). The MDA is one of the main intermediates between lipid peroxidation and oxidative stress (Nielsen et al., 1997). It was measured by HPLC, a sensitive and specific method to determine the MDA molecule itself and not other related aldehyde compounds. Even though the level of oxidative stress cannot be based on ROM and MDA concentrations alone (Halliwell and Chirico, 1993), measurements on hydroperoxides and lipid peroxidation have been used as indicators of oxidative stress (Nielsen et al., 1997). 

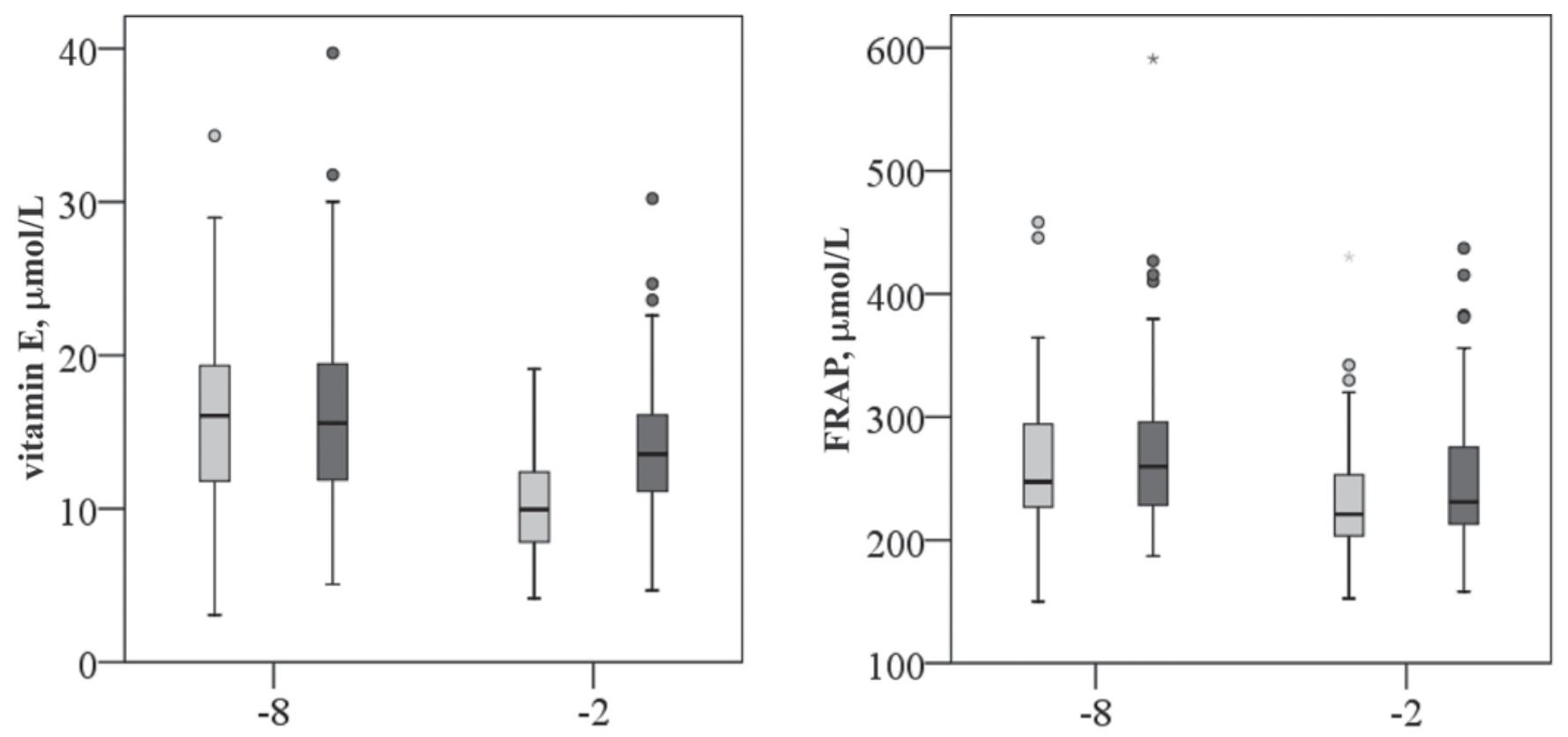

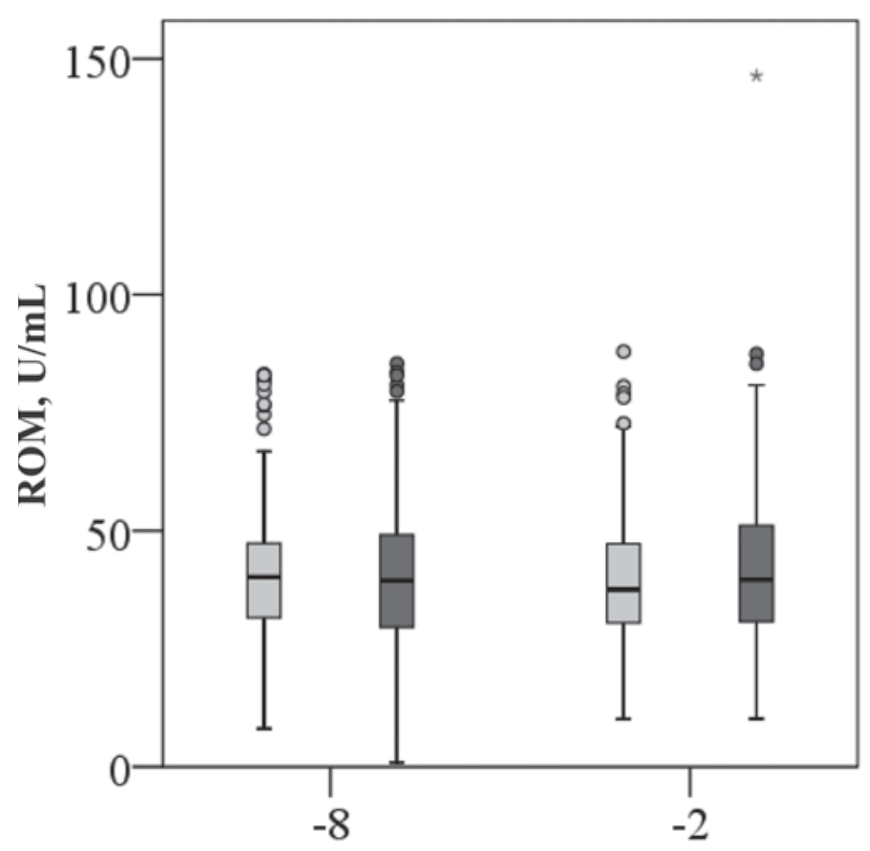

Weeks post partum

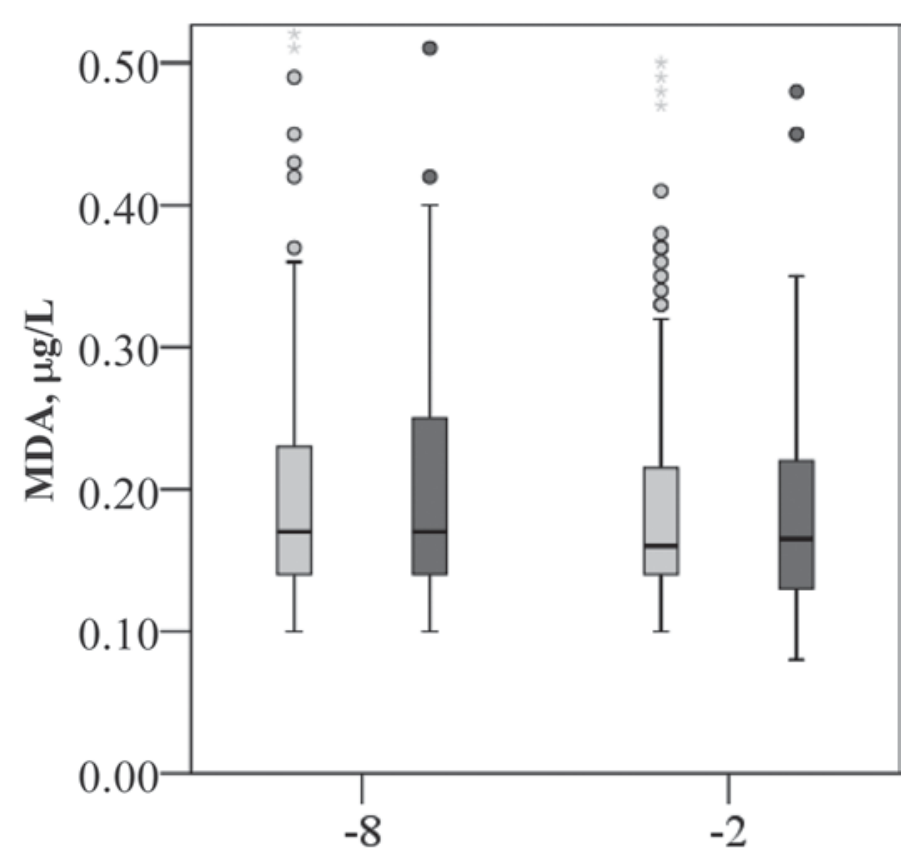

Weeks post partum

Figure 2. Blood $\alpha$-tocopherol, ferric-reducing ability of plasma assay (FRAP), malondialdehyde (MDA), and reactive oxygen metabolites (ROM) in the control (light gray box plots) and supplemented groups (dark gray box plots) during the dry period. Medians and quartiles are displayed in the box. Upper and lower bars represent 97.5 and 2.5 percentiles, respectively. Point markers indicate outliers, and asterisks indicate extreme outliers.

Although beneficial effects of vitamin $\mathrm{E}$ on the health of dairy cows are often attributed to reduced oxidative stress, oxidant parameters have rarely been measured in dairy cows supplemented with vitamin E. Most reports cover periparturient (metabolic) changes and oxidative stress levels around calving (Brzezinska-Slebodzinska et al., 1994; Bernabucci et al., 2005; Castillo et al., 2005). For example, Bernabucci et al. (2005) observed a drop in ROM levels around calving. Changes in blood concentrations of MDA or precursors of MDA (thio- 
Table 3. Results of Student's t-tests on reactive oxygen metabolites (ROM) and malondialdehyde (MDA) at 2 wk antepartum between vitamin E-supplemented $(3,000 \mathrm{IU} / \mathrm{d})$ and control $(135 \mathrm{IU} / \mathrm{d})$ groups within the 8 retrospectively defined subgroups

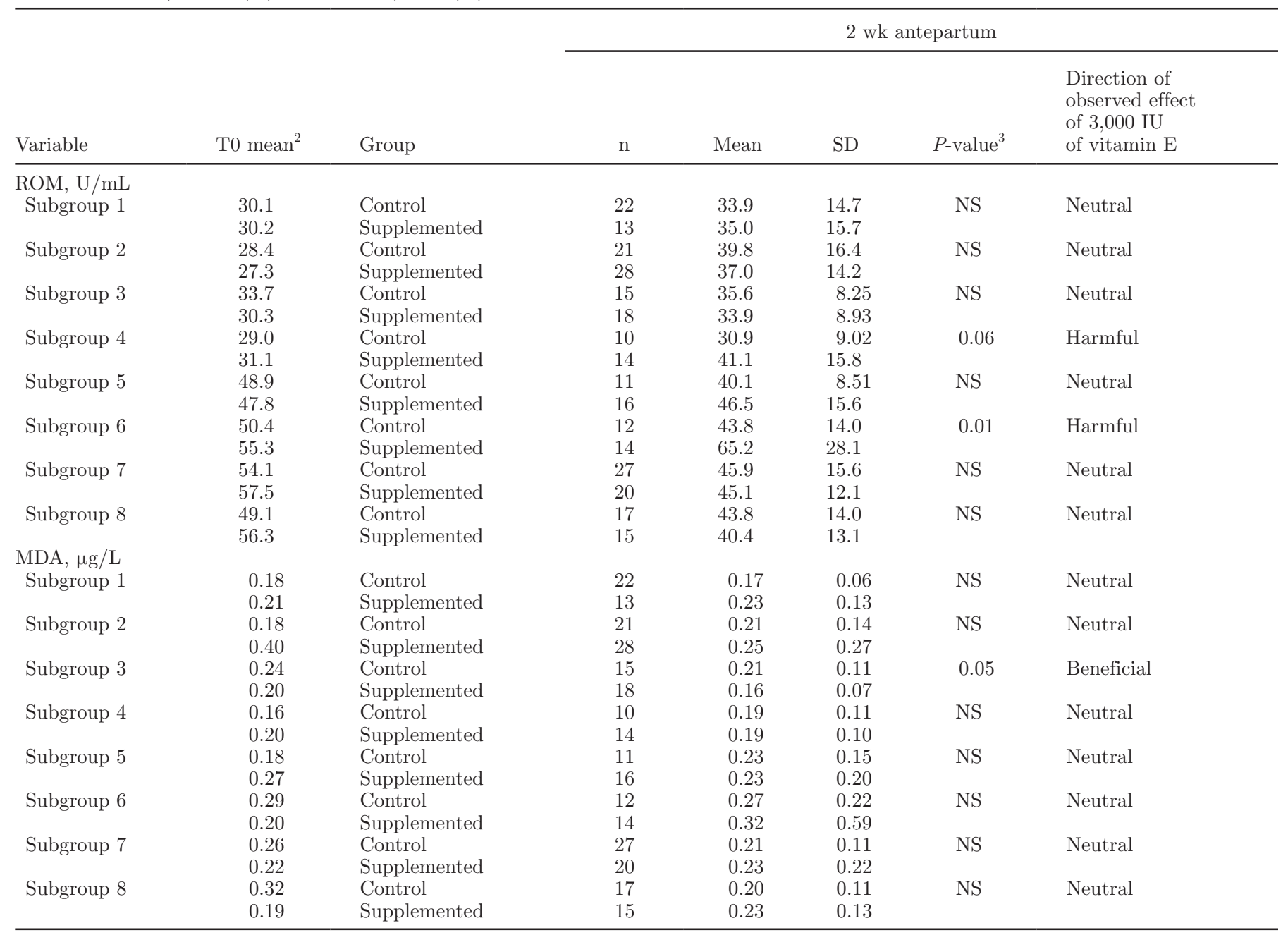

${ }^{1}$ Displayed are the number of cows per group within subgroup, mean level of ROM and MDA at dry off (T0) and 2 wk antepartum, and $P$-values of difference between the supplemented and control group at $2 \mathrm{wk}$ antepartum and interpretation.

${ }^{2}$ No significant differences between supplemented and control group within the 8 subgroups at T0.

${ }^{3}$ Statistical tests were carried out on $\operatorname{logROM}$ and $\log \mathrm{MDA}$ values.

barbituric acid reactive substances) were observed in periparturient cows (Bernabucci et al., 2005; Castillo et al., 2005). We hypothesize that a difference exists between acute oxidative stress and chronic oxidative stress based on the duration of high levels of free radicals in the body. Chronic oxidative stress has been described for chronic diseases such as diabetic or addictive smoking (Young and Woodside, 2001). In such chronic conditions, constant production of free radicals might occur relative to that in healthy bodies. Acute oxidative stress is expressed as a sudden increase in free radicals due to, for example, parturition. Normally the body would be able to detoxify the free radicals very quickly. High vitamin E supplementation in combination with insufficient VERS might give rise to chronic oxidative stress. If high levels of free radicals persist in the whole body for a longer period, we hypothesize that cell membranes of immune cells may be damaged. This might lead to less functional immune cells, and the body might be more sensitive for diseases (Frei et al., 1988; Young and Woodside, 2001). In our study, ROM and MDA levels were defined $2 \mathrm{wk}$ antepartum to avoid temporal and short-lived changes due to parturition and to better determine the chronic effect of vitamin E supplementation during the dry period on ROM and MDA levels with subsequent effect on mastitis risk postpartum.

In this research, 3 parameters were used to determine the VERS status: FRAP, GSHpx, and the GSH:GSSG ratio. Although not all possible parameters from Nwose 


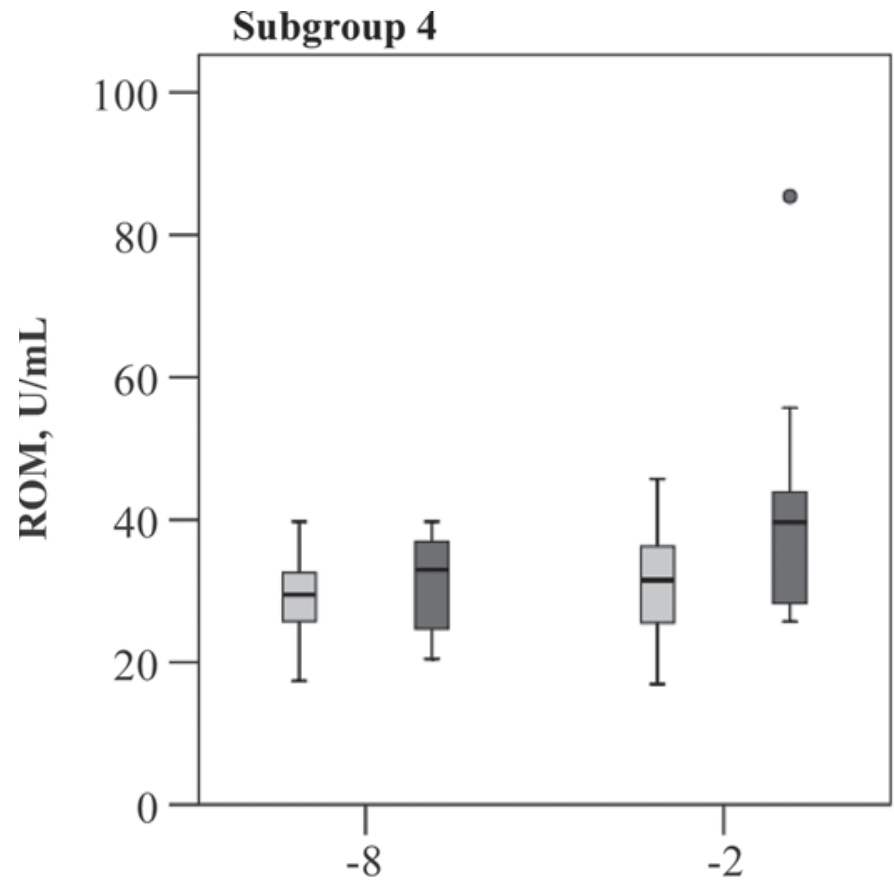

Weeks post partum

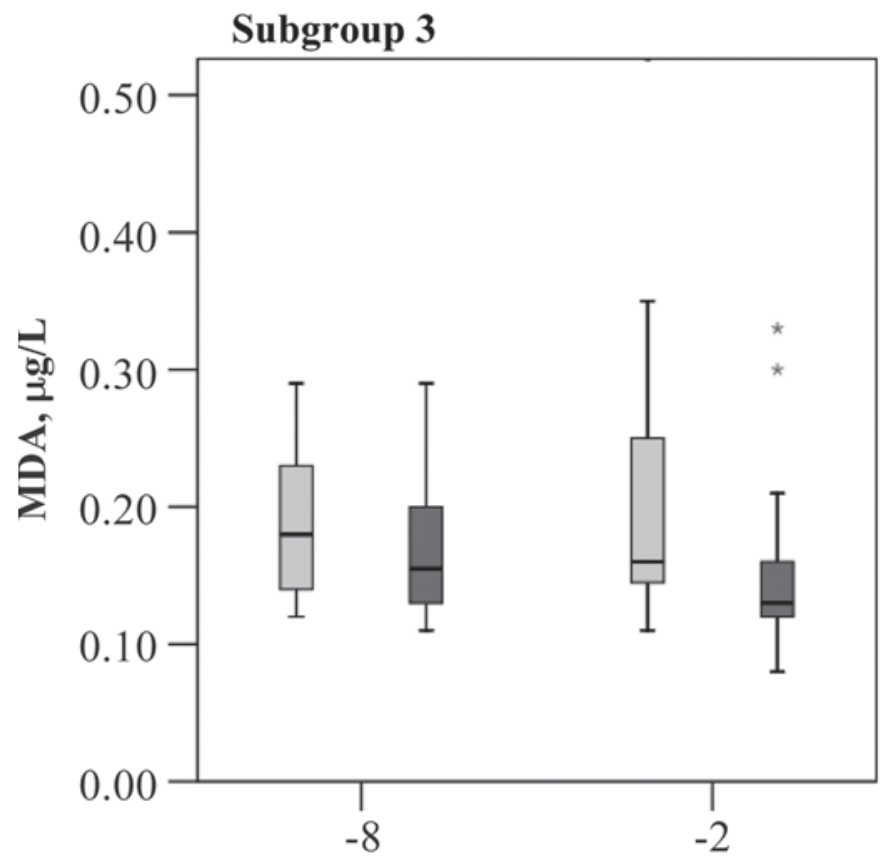

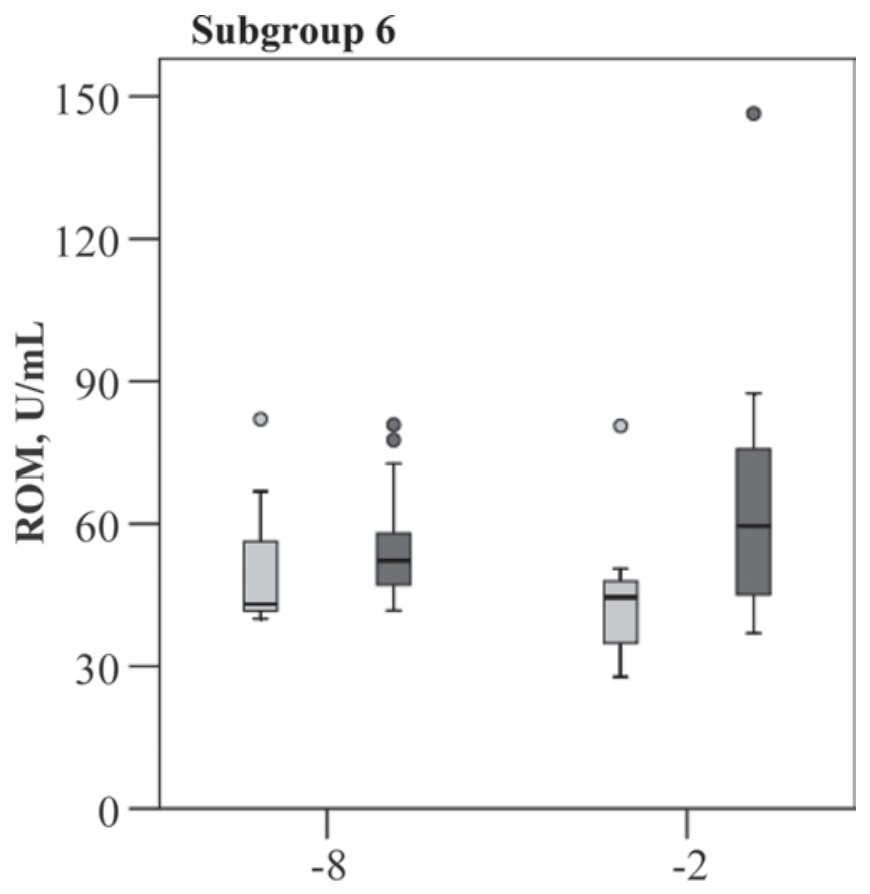

Weeks post partum

\section{Weeks post partum}

Figure 3. Blood reactive oxygen metabolites (ROM) in the control (light gray box plots) and supplemented groups (dark gray box plots) during the dry period for subgroup 4 (cows without oxidative stress, a high vitamin E status, and an insufficient vitamin E regeneration system (VERS) at T0; upper left figure) and subgroup 6 (cows with oxidative stress, not a high vitamin E status, and an insufficient VERS at T0; upper right figure), and blood malondialdehyde (MDA) in the control (light gray box plots) and supplemented groups (dark gray box plots) during the dry period for subgroup 3 (cows without oxidative stress, a high vitamin E status, and a sufficient VERS at T0; lower left figure). Medians and quartiles are displayed in the box. Upper and lower bars represent 97.5 and 2.5 percentiles, respectively. T0 $=$ dry off. 
Table 4. Descriptives of numbers of eventually healthy and eventually mastitic cows at 2 wk antepartum (n) and mean level of reactive oxygen metabolites (ROM) and malondialdehyde (MDA) ${ }^{1}$

\begin{tabular}{llrcc}
\hline Group & Cows & $\mathrm{n}$ & Mean & $\mathrm{SD}$ \\
\hline Control ROM, U/mL & Healthy & 119 & 39.6 & 14.7 \\
Supplemented ROM, U/mL & Mastitis & 16 & 41.6 & 11.6 \\
& Healthy & 115 & 41.1 & 15.1 \\
Control MDA, $\mu \mathrm{g} / \mathrm{L}$ & Mastitis & 23 & 48.2 & 26.7 \\
& Healthy & 119 & 0.21 & 0.11 \\
Supplemented MDA, $\mu \mathrm{g} / \mathrm{L}$ & Mastitis & 16 & 0.24 & 0.19 \\
& Healthy & 115 & 0.21 & 0.17 \\
& Mastitis & 23 & 0.34 & 0.49 \\
\hline
\end{tabular}

${ }^{1}$ The differences between mean level of ROM and MDA (tested with Student's $t$-tests) in eventually healthy and eventually mastitic cows at 2 wh antepartum were nonsignificant. Statistical tests were carried out on $\log \mathrm{ROM}$ and $\log \mathrm{MDA}$ values.

et al. (2008) were used to calculate the VERS status of the cows, the GSH:GSSG ratio, GSHpx, and FRAP should give a good indication. To properly calculate the VERS status glutathione, vitamin C and co-enzyme Q10 should have been analyzed as well.

To determine the VERS status, reference values of co-antioxidants of the oxidative stress cascade were needed. Unfortunately, in dairy cows, information about these reference values is lacking. To calculate for the 3 physiological states (oxidative stress, vitamin E status, and VERS), we decided to use the median values for ROM and vitamin E, the 20th percentiles for FRAP and GSHpx, and the 80th percentile for the GSH:GSSG ratio at $\mathrm{T} 0$ as cut-off values. The frequency distributions of ROM and logMDA at 2 wk antepartum both showed a bimodal distribution (results not shown), suggesting the existence of 2 separate cow populations. We chose the 80th percentile as the cut-off value between the normal and high subpopulation.

The hypothesis described by Nwose et al. (2008) was supported in our study, although cut-off values were based on the above data-driven assumptions. The increased level of ROM measured in subgroups 4 and 6 at 2 wk antepartum might translate into a contraindication for vitamin $\mathrm{E}$ supplementation in some individual cows during the dry period. In the present study, 3,000 IU of vitamin $\mathrm{E} / \mathrm{d}$ could overload the oxidative stress cascade with vitamin E. Vitamin E supplementation did increase oxidative stress in some subgroups of cows with an insufficient VERS (subgroup 4 and 6) instead of the expected effect that vitamin E would lead to reduced oxidative stress antepartum. From the 3 physiological states (oxidative stress, vitamin E, and VERS status) used in this study, VERS status seemed to be most crucial in the decision of whether to supplement. An explanation for the fact that our results did not completely follow the theoretically expected effects of the hypothetical decision tree (Figure 1) might be the difference in level of oxidative stress in humans and cows. Humans with chronic diseases may suffer from a continuous high level of oxidative stress, whereas in dairy cows, we try to prevent the increase of acute oxidative stress around parturition. In humans and in cows, vitamin E might be supplemented with for different reasons, to reduce existing oxidative stress or to prevent an increase of oxidative stress, respectively.

In general, free radicals cause cell damage and might influence the functions of immune cells (Politis et al., 2004; Sordillo and Aitken, 2009) resulting in increased mastitis risk. Indeed, our retrospective analysis found that cows that developed clinical mastitis in early lactation had higher levels of ROM and MDA 2 wk antepartum. Because the study design was not optimized for a comparison between mastitic and healthy cows, the total number of mastitic cows might have been too low to reach significance.

The above-described theory might also explain the variation in results of the different vitamin $\mathrm{E}$ studies and our unexpected adverse results (Bouwstra et al., 2010). The degree of oxidative stress, the initial vitamin E level, and the VERS status of the animals in these older studies might have been different in comparison with more recent studies that showed no or adverse effect of vitamin E supplementation on udder health (Bouwstra et al., 2010). Although time trends and differences in vitamin $\mathrm{E}$ between groups are in agreement with others, the mean vitamin $\mathrm{E}$ level at dry off of the cows used in this experiment was 2 to 3 times higher than starting levels of cows in other reports (Weiss et al., 1990b; LeBlanc et al., 2002). The high vitamin $\mathrm{E}$ level at dry off seems the most reasonable explanation for the adverse results of the present study. Vitamin E supplementation might only show positive effects in studies where cows started with a marginal or deficient vitamin E status, which was then improved after vitamin E supplementation, and where there was sufficient capacity to regenerate $\alpha$-tocopheroxyl radicals. 


\section{CONCLUSIONS}

Not every dry dairy cow needs vitamin E supplements. Specifically, to advise extra vitamin E supplements during the dry period without knowing the prior vitamin E status, the prior VERS status, and the prior level of oxidative stress might be hazardous.

\section{ACKNOWLEDGMENTS}

This study is part of the 5-yr mastitis program of the Dutch Udder Health Centre and was financially supported by the Dutch Dairy Board. The authors acknowledge T. J. G. M. Lam (Deventer, the Netherlands) and Y. H. Schukken (Ithaca, NY) for critical comments.

\section{REFERENCES}

Benzie, I. F., and J. J. Strain. 1996. The ferric reducing ability of plasma (FRAP) as a measure of "antioxidant power": The FRAP assay. Anal. Biochem. 239:70-76.

Bernabucci, U., B. Ronchi, N. Lacetera, and A. Nardone. 2005. Influence of body condition score on relationships between metabolic status and oxidative stress in periparturient dairy cows. J. Dairy Sci. 88:2017-2026

Bouwstra, R. J., M. Nielen, J. A. Stegeman, P. Dobbelaar, J. R. Newbold, E. H. J. M. Jansen, and T. van Werven. 2010. Vitamin E supplementation during the dry period in dairy cattle. Part I: Adverse effect on incidence of mastitis postpartum in a double-blind randomized field trial. J. Dairy Sci. 93:5684-5695.

Brigelius-Flohé, R. 2007. Adverse effects of vitamin E by induction of drug metabolism. Genes Nutr. 2:249-256.

Brzezinska-Slebodzinska, E., J. K. Miller, J. D. Quigley, III, J. R. Moore, and F. C. Madsen. 1994. Antioxidant status of dairy cows supplemented prepartum with vitamin E and selenium. J. Dairy Sci. $77: 3087-3095$

Castillo, C., J. Hernandez, A. Bravo, M. Lopez-Alonso, V. Pereira, and J. L. Benedito. 2005. Oxidative status during late pregnancy and early lactation in dairy cows. Vet. J. 169:286-292.

Frei, B., R. Stocker, and B. N. Ames. 1988. Antioxidant defenses and lipid peroxidation in human blood plasma. Proc. Natl. Acad. Sci. USA 85:9748-9752.

Girotti, A. W. 1998. Lipid hydroperoxide generation, turnover, and effector action in biological systems. J. Lipid Res. 39:1529-1542.

Goff, J. P., and J. R. Stabel. 1990. Decreased plasma retinol, alpha-tocopherol, and zinc concentration during the periparturient period: Effect of milk fever. J. Dairy Sci. 73:3195-3199.

Halliwell, B., and S. Chirico. 1993. Lipid peroxidation: Its mechanism, measurement, and significance. Am. J. Clin. Nutr. 57(5 Suppl.):715S-724S; discussion 724S-725S.

Ho, C. T., and A. C. Chan. 1992. Regeneration of vitamin E in rat polymorphonuclear leucocytes. FEBS Lett. 306:269-272.

Ibrahim, W., U. S. Lee, C. C. Yeh, J. Szabo, G. Bruckner, and C. K. Chow. 1997. Oxidative stress and antioxidant status in mouse liver: Effects of dietary lipid, vitamin E and iron. J. Nutr. 127:14011406

LeBlanc, S. J., T. F. Duffield, K. E. Leslie, K. G. Bateman, J. TenHag, J. S. Walton, and W. H. Johnson. 2002. The effect of prepartum injection of vitamin $\mathrm{E}$ on health in transition dairy cows. J. Dairy Sci. 85:1416-1426.
LeBlanc, S. J., T. H. Herdt, W. M. Seymour, T. F. Duffield, and K. E. Leslie. 2004. Peripartum serum vitamin E, retinol, and betacarotene in dairy cattle and their associations with disease. J. Dairy Sci. 87:609-619.

Miller, E. R. III, R. Pastor-Barriuso, D. Dalal, R. A. Riemersma, L. J. Appel, and E. Guallar. 2005. Meta-analysis: High-dosage vitamin E supplementation may increase all-cause mortality. Ann. Intern. Med. 142:37-46.

Miller, J. K., E. Brzezinska-Slebodzinska, and F. C. Madsen. 1993 Oxidative stress, antioxidants, and animal function. J. Dairy Sci. $76: 2812-2823$.

Mosca, L., C. L. Banka, E. J. Benjamin, K. Berra, C. Bushnell, R. J. Dolor, T. G. Ganiats, A. S. Gomes, H. L. Gornik, C. Gracia, M. Gulati, C. K. Haan, D. R. Judelson, N. Keenan, E. Kelepouris, E. D. Michos, L. K. Newby, S. Oparil, P. Ouyang, M. C. Oz, D. Petitti, V. W. Pinn, R. F. Redberg, R. Scott, K. Sherif, S. C. Smith Jr., G. Sopko, R. H. Steinhorn, N. J. Stone, K. A. Taubert, B. A. Todd, E. Urbina, and N. K. Wenger. 2007. Evidence-based guidelines for cardiovascular disease prevention in women: 2007 update. J. Am. Coll. Cardiol. 49:1230-1250.

Nielsen, F., B. B. Mikkelsen, J. B. Nielsen, H. R. Andersen, and P. Grandjean. 1997. Plasma malondialdehyde as biomarker for oxidative stress: Reference interval and effects of life-style factors. Clin. Chem. 43:1209-1214.

Nwose, E. U., H. F. Jelinek, R. S. Richards, and P. G. Kerr. 2008. The 'vitamin E regeneration system' (VERS) and an algorithm to justify antioxidant supplementation in diabetes - A hypothesis. Med. Hypotheses 70:1002-1008.

Persson Waller, K. P., C. Hallén Sandgren, U. Emanuelson, and S. K. Jensen. 2007. Supplementation of $R R R$ - $\alpha$-tocopheryl acetate to periparturient dairy cows in commercial herds with high mastitis incidence. J. Dairy Sci. 90:3640-3646.

Politis, I., I. Bizelis, A. Tsiaras, and A. Baldi. 2004. Effect of vitamin E supplementation on neutrophil function, milk composition and plasmin activity in dairy cows in a commercial herd. J. Dairy Res. 71:273-278.

Sen, C. K., M. Atalay, and O. Hanninen. 1994. Exercise-induced oxidative stress: Glutathione supplementation and deficiency. J. Appl. Physiol. 77:2177-2187.

Smith, K. L., J. H. Harrison, D. D. Hancock, D. A. Todhunter, and H. R. Conrad. 1984. Effect of vitamin e and selenium supplementation on incidence of clinical mastitis and duration of clinical symptoms. J. Dairy Sci. 67:1293-1300.

Sordillo, L. M., and S. L. Aitken. 2009. Impact of oxidative stress on the health and immune function of dairy cattle. Vet. Immunol. Immunopathol. 128:104-109.

Thrusfield, M., C. Ortega, I. de Blas, J. P. Noordhuizen, and K. Frankena. 2001. Win episcope 2.0: Improved epidemiological software for veterinary medicine. Vet. Rec. 148:567-572.

Weiss, W. P., J. S. Hogan, K. L. Smith, and K. H. Hoblet. 1990a. Relationships among selenium, vitamin e, and mammary gland health in commercial dairy herds. J. Dairy Sci. 73:381-390.

Weiss, W. P., D. A. Todhunter, J. S. Hogan, and K. L. Smith. 1990b. Effect of duration of supplementation of selenium and vitamin $\mathrm{E}$ on periparturient dairy cows. J. Dairy Sci. 73:3187-3194.

Winterbone, M. S., M. Sampson, S. Saha, J. Hughes, and D. Hughes. 2007. Pro-oxidant effect of alpha-tocopherol in patients with Type 2 Diabetes after an oral glucose tolerance test- A randomised controlled trial. Cardiovasc. Diabetol. 6:8.

Yamanaka, G., H. Kawashima, Y. Suganami, C. Watanabe, Y. Watanabe, T. Miyajima, K. Takekuma, S. Oguchi, and A. Hoshika. 2006. Diagnostic and predictive value of CSF d-ROM level in influenza virus-associated encephalopathy. J. Neurol. Sci. 243:71-75.

Young, I. S., and J. V. Woodside. 2001. Antioxidants in health and disease. J. Clin. Pathol. 54:176-186. 